\title{
Caracterização dos acidentes perfurocortantes de acadêmicos e profissionais de uma instituição de ensino superior e saúde do sul do país: estudo transversal
}

\author{
Characterization of needlestick injuries of academics and professionals from a higher \\ education and health institution in the south of the country: a cross-sectional study
}

Caracterización de lesiones por pinchazos de agujas en académicos y profesionales de una institución de educación superior y salud en el sur del país: un estudio transversal

Ana Maria Silveira dos Santos Galarça ${ }^{1 *}$, Camila Thurow Becker ${ }^{1}$, Rafael Guerra Lund ${ }^{1}$, Adrize Rutz Porto ${ }^{1}$, Diana Cecagno'.

\begin{abstract}
RESUMO
Objetivo: Descrever a prevalência dos acidentes por material biológico e/ou perfurocortante em uma Faculdade de Odontologia da região Sul do Brasil por período de doze meses. Métodos: Qualitativo, observacional, do tipo descritivo, tendo am ostragem por conveniência, sendo os dados analisados por estatística descritiva. Resultados: Verificou-se que todos os acidentes ocorreram com acadêmicos (100\%), sendo a maioria entre o sexo feminino (87\%), durante as clínicas da graduação $(39,1 \%)$; o instrumental mais recorrente foi a seringa Carpule (32\%), causando as ocorrências durante os atendimentos $(78,3 \%)$, embora a maioria relatasse fazer uso do equipamento de proteção individual ( $82,6 \%)$. Não houve contaminação em nenhum caso (100\%), mesmo com pacientes fonte com resultados positivos para Hepatite C (20\%) e vírus da imunodeficiência humana $(13,3 \%)$ que receberam atendimentos odontológicos. Entre as ações de recomendação estão a profilaxia pós-exposição $(21,7 \%)$ e reforço vacinal $(47,8 \%)$, sendo que em alguns casos não foi necessário nenhum procedimento $(30,4 \%)$. Conclusão: Existem muitos casos de subnotificação na Odontologia, seja por motivos de burocracia, ou outro. Isso leva ao aumento dos riscos envolvidos em acidentes perfurocortantes. Estimula-se a ação conjunta de setores da Instituição para reforçar a importância da biossegurança e da notificação de acidentes com exposição aos agentes biológicos.
\end{abstract}

Palavras-chave: Acidentes com perfurocortantes, Contenção de riscos biológicos, Odontólogos.

\section{ABSTRACT}

Objective: To describe the prevalence of accidents caused by biological and/or sharps in a Dental School in southem Brazil for a period of twelve months. Methods: Qualitative, observational, descriptive, with convenience sampling, with data analyzed using descriptive statistics. Results: It was found that all accidents occurred with academics (100\%), the majority among females (87\%), during undergraduate clinics (39.1\%); the most recurrent instrument was the Carpule syringe (32\%), causing occurrences during consultations $(78.3 \%)$, although the majority reported using personal protective equipment $(82.6 \%)$. There was no contamination in any case $(100 \%)$, even with source patients with positive results for Hepatitis C (20\%) and human immunodeficiency virus (13.3\%) who received dental care. Among the recommended actions are post-exposure prophylaxis $(21.7 \%)$ and vaccine booster $(47.8 \%)$, and in some cases no procedure was necessary (30.4\%). Conclusion: There are many cases of underreporting in Dentistry, whether due to bureau cracy or other reasons. This leads to increased risks involved in sharps accidents. The joint action of sectors of the Institution is encouraged to reinforce the im portance of biosafety and the notification of accidents with exposure to biological agents.

Key words: Accidents with sharps, Containment of biohazards, Dentists.

\section{RESUMEN}

Objetivo: Describir la prevalencia de accidentes causados por biológicos y/o cortopunzantes en una Facultad de Odontología del sur de Brasil durante un período de doce meses. Métodos: Cualitativo, observacional, descriptivo, con muestreo por conveniencia, con datos analizados mediante estadística descriptiva. Resultados: Se constató que todos los accidentes ocurrieron con académicos (100\%), la mayoría entre mujeres (87\%), durante clínicas de pregrado $(39,1 \%)$; el instrumento más recurrente fue la jeringa Carpule (32\%), provocando ocurrencias durante las consultas (78,3\%), aunque la mayoría refirió utilizar equipo de protección personal (82,6\%). No hubo contaminación en ningún caso (100\%), incluso con pacientes fuente con resultado positivo para Hepatitis C $(20 \%)$ y virus de la inmunodeficiencia humana $(13,3 \%)$ que recibieron atención odontológica. Entre las acciones recomendadas están la profilaxis postexposición ( $21,7 \%)$ y el refuerzo vacunal $(47,8 \%)$, y en algunos casos no fue necesario ningún procedimiento $(30,4 \%)$. Conclusión: Existen muchos casos de subregistro en Odontología, ya sea por burocracia u otras razones. Esto conduce a mayores riesgos involucrados en accidentes cortopunzantes. Se fomenta la acción conjunta de sectores de la Institución para reforzar la importancia de la bioseguridad y la notificación de accidentes con exposición a agentes biológicos.

Palabras clave: Accidentes con objetos cortopunzantes, Contención de riesgos biológicos, Odontólogos.

${ }^{1}$ Universidade Federal de Pelotas (UFPel), Pelotas - RS. *E-mail:anamariagalarca@gmail.com 


\section{INTRODUÇÃO}

A biossegurança na área da saúde consiste em um conjunto de normas, ações e procedimentos considerados seguros para prevenir, controlar, minimizar ou eliminar riscos à saúde e ao meio ambiente. Dentre as profissões com maior exposição ao risco de contaminação, destaca-se a Odontologia, pois os profissionais estão rotineiramente em contato com sangue e saliva, considerados possíveis agentes biológicos infecciosos (PINHEIRO SL, et al., 2016; MAZZUTI WJ, et al., 2018).

Portanto, algumas medidas de prevenção básicas devem ser seguidas pelo Cirurgião Dentista (CD). Dentre elas: desinfecção de bancadas e equipamentos, esterilização dos instrumentais, higienização adequada das mãos e cronograma de vacinação atualizado. Além do uso de equipamentos de proteção individual (EPI), pois propiciam uma cadeia asséptica e diminuem riscos de contaminação cruzada (CARVALHO MJA e OLIVEIRA AHA 2015; MARTINS JR, et al., 2020).

A adoção de condutas de biossegurança é imprescindível na rotina clínica, visto que, em ambientes odontológicos existem riscos ergonômicos, químicos, físicos e biológicos. Estes acabam por exporo cirurgião dentista e sua equipe a doenças infectocontagiosas. Além disso, as precauções devem ser universais, ou seja, adotadas independentemente do diagnóstico confirmado ou não, de qualquer doença relatada pelo usuário do serviço de odontologia (MINISTÉRIO DA SAÚDE 2015; OLIVEIRA A, et al., 2018).

Independentemente da condição do paciente, existem medidas que devem ser adotadas pelo cirurgião dentista. Por exemplo: desinfecção de bancadas e equipamentos, esterilização dos instrumentais, higienização adequada das mãos e cronograma de vacinação atualizado. Além do uso de equipamentos de proteção individual, pois propiciam uma cadeia asséptica que diminuem riscos de contaminação cruzada (MINISTÉRIO DA SAÚDE, 2010; OLIVEIRA RHG e ALMEIDA T, 2015).

Dentre os EPls necessários estão o uso de máscara (recomenda-se N95 ou PFF2), gorro, luva, avental, óculos e protetor facial tanto para sua proteção quanto do paciente. Assim como a esterilização dos instrumentais utilizados, e a desinf ecção dos equipamentos com álcool $70 \%$ entre uma consulta e outra, tais como: cadeiras, bancadas e outras peças que ficam expostas a aerossóis com fluidos biológicos (YOUNES T, et al., 2017; MONTALLI VAM, et al., 2020).

Além do Equipamento de Proteção Individual (EPI), aspectos de higiene pessoal e organização do ambiente de trabalho são medidas importantes, uma vez que influenciam na qualidade e na segurança do trabalho. Ressalta-se a importância de proteger o paciente durante os atendimentos, uma vez que a manipulação de instrumentais pontiagudos, produtos químicos e outros materiais contaminados podem causar graves acidentes (NOGUEIRA SA, et al., 2016; YOUNES T, et al., 2017).

Também é necessário proteger o paciente durante os atendimentos, uma vez que a manipulação de instrumentais pontiagudos, produtos químicos e outros materiais contaminados podem causar acidentes. A não adoção desses princípios, na sua totalidade ou em parte, porqualquer membro da equipe de atendimento,

O consultório odontológico é um ambiente rico em microrganismos que podem ser transmitidos por sangue e fluídos orgânicos. Ocorrem por contato direto com os pacientes contaminados ou por via aérea, através de partículas em suspensão, como no espirro e nas gotículas suspensas no ambiente (YOUNES T, et al., 2017).

Durante $o$ atendimento clínico, a contaminação cruzada pode ser causada pela disseminação de respingos e aerossóis carregados de patógenos oriundos das seringas tríplices, turbinas de alta rotação e outros instrumentais perfurantes e cortantes que são utilizadas em procedimentos odontológicos (GAMA TM, et al., 2020).

Risco biológico envolve bactérias, vírus, protozoários e fungos que atuam como agentes de transmissão, sendo o consultório odontológico um ambiente rico desses microrganismos. É preciso que todos os indivíduos sejam atendidos criteriosamente, independentemente da situação sistêmica. Por isso, a vacinação do cirurgião-dentista torna-se de extrema importância, como é o caso da vacina contra hepatite $\mathrm{B}$, que induz imunidade em 90 a 95\% dos casos (MINISTÉRIO DA SAÚDE, 2008; MINISTÉRIO DA SAÚDE, 2013; FERREIRA LQ, et al., 2018). 
Acidentes com perfurocortantes são os mais comuns na Odontologia e acontecem desde a formação profissional dos cirurgiões-dentistas e muitos acontecem durante a desinfecção e a lavagem dos instrumentais. A negligência no uso dos EPls completos são fatores decisivos para que ocorram contaminações (principalmente em relação aos Vírus da Imunodeficiência Humana (HIV), Hepatite B (HBV) e Hepatite C (HCV) (CUNHA QB, et al., 2017; MAZZUTTI WJ, et al., 2018; MARTINS JR, et al., 2020).

Apesar de ainda não serem considerados profissionais, os acadêmicos dos cursos de Odontologia, prestam cuidados em saúde bucal diariamente nas clínicas de suas instituições de ensino e enfrentam os riscos provenientes à profissão (ARANTES DC, et al., 2015; GALARÇA AMSS e LUND RG, 2020).

Estudos relatam que muitas vezes os alunos não dão a devida importância aos acidentes, encarando -os com naturalidade, deixando de fazer a notificação e tão pouco procurando orientações e ajuda necessária (TREZENA S, et al., 2020). Tais atitudes podem gerar agravos à saúde do acidentado. Por isso, incentiva-se a adoção de medidas que favoreçam o conhecimento das condutas e os riscos em casos de acidentes nas clínicas odontológicas (MAZZUTTI WJ, et al., 2018).

Percebe-se que os cirurgiões-dentistas se consideram informados sobre as formas de contaminação e prevenção de riscos. Ao mesmo tempo, demonstram um conhecimento limitado. Logo, é necessário que sejam realizadas campanhas de divulgação sobre políticas públicas, enfatizando a importância das normas de biossegurança entre profissionais da saúde (CARVALHO MJA e OLIVEIRA AHA. 2015; OLIVEIRA A, et al., 2018).

Os acidentes de trabalho com exposições a materiais biológicos ocorrem frequentemente entre os trabalhadores da saúde no mundo inteiro. As principais causas são os materiais utilizados, ambientes insalubres e/ou as condições em que o trabalho é executado (COUTO PLS, et al., 2018).

Nesse contexto, o presente estudo teve como objetivo retratar a predominância de ocorrências provenientes de material biológico e/ou perfurocortante entre odontólogos e discentes de uma Faculdade do Sul do Brasil, por período de doze meses.

\section{MÉTODOS}

Trata-se de um estudo qualitativo Estudo tipo documental, transversal e descritivo realizado com amostragem por conveniência. A pesquisa documental corresponde a origem da coleta de dados de documentos, caracterizados por fontes primárias.

Estas podem ser feitas no momento em que o fato ou fenômeno ocorrem, ou depois. O delineamento deste estudo é do tipo transversal, pois mediu a prevalência e analisou as frequências de fatores de risco de determinado desfecho (NEDEL WL e SILVEIRA F, 2016).

Foram incluídos discentes, docentes e profissionais que exerceram atividades em clínica odontológica, e que tenham sido expostos a material biológico e/ou acidente perfurocortante, durante o período de fevereiro de 2019 a fevereiro de 2020, atendidos no serviço de testagem rápida Municipal e/ou no Serviço de atendimento a acidentes ocupacionais de uma Faculdade de Odontologia da região Sul do Brasil.

Os indivíduos que passaram por acidentes perfurocortantes foram esclarecidos e informados sobre os objetivos desta pesquisa e incluídos na mesma mediante a assinatura do Termo de Consentimento Livre e Esclarecido (TCLE), o qual foi feito em duas vias. Foram excluídos todos aqueles que sof reram acidentes nas clínicas odontológicas, mas que não tinham registro de atendimento nos serviços.

Análise de dados foi feita por meio de estatística descritiva, com apresentação em tabelas de frequências absolutas e relativas. Este estudo foi aprovado pelo Comitê de Ética em Pesquisa da Faculdade de Odontologia da UFPel (Protocolo CAAE 40874414.6.0000.5317).

\section{RESULTADOS E DISCUSSÃO}

A partir dos dados obtidos nas fichas do serviço de testagem rápida Municipal e no Serviço de atendimento a acidentes ocupacionais de uma Faculdade de Odontologia da região Sul do Brasil, foram descritos na Tabela 1, os registros de acidentes perfurocortantes ocorridos nos primeiros doze meses deste estudo. 
Tabela 1 - Descrição dos dados referentes ao Protocolo Institucional da Unidade (formulário de acompanhamento de acidente com perfurocortantes).

\begin{tabular}{|c|c|c|}
\hline Variável & Frequência absoluta (n) & Frequência relativa (\%) \\
\hline \multicolumn{3}{|l|}{ Acidentado } \\
\hline Acadêmicos & 23 & 100 \\
\hline Professores & 0 & 0 \\
\hline Técnicos Terceirizados & 0 & 0 \\
\hline \multicolumn{3}{|l|}{ Sexo } \\
\hline Feminino & 20 & 87 \\
\hline Masculino & 3 & 13 \\
\hline \multicolumn{3}{|l|}{ Local/Disciplina } \\
\hline $\mathrm{CEOs}^{*}$ & 4 & 17,4 \\
\hline Disciplinas da graduação ** & 9 & 39,1 \\
\hline $\mathrm{ECOs}^{* \star \star}$ & 4 & 17,4 \\
\hline Pronto Atendimento & 2 & 8,7 \\
\hline Pós-graduação & 2 & 8,7 \\
\hline Projetos de extensão ${ }^{\star \star \star \star}$ & 2 & 8,7 \\
\hline \multicolumn{3}{|l|}{ Instrumental } \\
\hline Cureta periodontal & 4 & 21,0 \\
\hline Descolador de Molt & 2 & 10,5 \\
\hline Ponta diamantada & 2 & 10,5 \\
\hline Seringa Carpule & 6 & 32,0 \\
\hline Sondas exploradoras ${ }^{\star \star \star \star \star}$ & 4 & 21,0 \\
\hline Tesoura Íris & 1 & 5,0 \\
\hline \multicolumn{3}{|l|}{ Momento do acidente } \\
\hline Atendimento & 18 & 78,3 \\
\hline Embalagem & 1 & 4,3 \\
\hline Lavagem & 3 & 13 \\
\hline Secagem & 1 & 4,3 \\
\hline \multicolumn{3}{|l|}{ EPI's } \\
\hline Utilizava EPI completo & 19 & 82,6 \\
\hline Não utilizava EPI completo & 4 & 17,4 \\
\hline
\end{tabular}

Legenda: *CEOs (Centro de Especialidades Odontológicas); **Disciplinas da graduação (Unidade Clínica Odontológica le II, Unidade de Cirurgia Buco Maxilo Facial I, II e III); ${ }^{\star \star \star E C O s ~(E s t a ́ g i o ~ e m ~ C l i n i c a ~ O d o n t o l o ́ g i a ~}$ I e II); ${ }^{* \star * \star}$ Projetos de extensão (Gepeto e Odonto ao Vivo); ${ }^{* * * *}$ Sondas exploradoras ( ${ }^{\circ} 5$, n9 e milimetrada Willians). Fonte: Galarça AMSS, et al., 2021.

Dos locais envolvidos, os mais citados foram as clínicas da graduação, onde são realizados procedimentos críticos, mas em grande parte semicríticos, ou seja, não há invasão do sistema vascular.

Acredita-se que esses acidentes ocorram pelo fato de os alunos julgarem muitos procedimentos como sendo de baixo risco de contaminação. Isso gera maior desatenção e uso incorreto de EPls frente aos procedimentos (PAIVA SN, et al., 2017; OLIVEIRA A, et al.,2018).

Assim como no estudo de León FC, et al. (2019), o instrumental mais mencionado foi a seringa Carpule. Esta contém a agulha anestésica, e devido a prática incorreta de reencape, facilmente provoca lesões nos membros superiores principalmente quando ocorrem movimentos bruscos (MUSSI M e MARASEA DCC, 2016; NOGUEIRA SA, et al., 2016; MARTINS JR, et al., 2018).

Por vezes, a anestesia é um ato que causa estresse ao paciente, e isso pode interferir na segurança do operador no decorrer do atendimento. Já as curetas periodontais e sondas exploradoras corroboram com outros estudos justificando sua natureza cruenta pelo fato desses instrumentais serem altamente afiados (MUSSI M e MARASEA DCC, 2016; PAIVA SN, et al., 2017; MAZZUTTI WJ, et al., 2018).

Grande parte dos acidentes ocorreram durante o atendimento, considerado um fator de tensão devido a relação que é estabelecida com o paciente (OLIVEIRA et al., 2018). Além do aluno estar sob constante avaliação dos professores, a pressa, a complexidade dos procedimentos, desorganização e até mesmo um movimento inesperado do paciente, podem causar acidente (LIMA AVM, et al., 2016; PAIVA SN, et al., 2017). 
Os dados observados assemelham-se em parte com estudos de mesmo enfoque em instituições de ensino odontológico, onde grande parte dos relatos são de exposição percutânea, em sua maioria durante procedimentos clínicos e cirúrgicos (PAIVA SN, et al., 2017). Além disso, 4 (17,4\%) acidentes ocorreram devido à falta dos óculos de proteção, não mencionados na variável Instrumental, por não serem classificados como acidentes perfurocortantes.

Outro momento crítico é na lavagem dos instrumentais, sendo recomendado o acréscimo de luvas de borracha para lavagem e secagem destes. Os alunos acidentados relataram o uso de luvas de procedimento no momento do acidente, porém, estas protegem contra leves injúrias e não impedem perfurações (OLIVEIRA A, et al., 2018; MAZZUTTI WJ, et al., 2018).

De acordo com a Tabela 2, todos os acidentados realizaram testes rápidos. Quanto aos pacientes fonte, apenas $15(65,2 \%)$ destes foram testados. Já dos outros indivíduos não há informações, pois, em muitos casos, já obtinham a liberação por término do procedimento, quando os alunos registraram os acidentes. Acredita-se que possa haver certo constrangimento em relatar ao paciente o ocorrido e ter que submeter 0 mesmo aos exames pós-exposição (LIMA AVM, et al., 2016).

Tabela 2 - Descrição dos dados referentes ao Protocolo Institucional da Unidade (Planilha de segmento de testagem rápida e vacinação).

\begin{tabular}{|c|c|c|}
\hline $\begin{array}{r}\text { Variável } \\
\end{array}$ & Frequência absoluta (n) & Frequência relativa (\%) \\
\hline \multicolumn{3}{|c|}{ Exames realizados pelos Acidentados } \\
\hline Realizou & 23 & 100 \\
\hline Não realizou & 0 & 0 \\
\hline \multicolumn{3}{|c|}{ Exames realizados pelos pacientes (Fonte) } \\
\hline Testes Rápidos & 15 & 65,2 \\
\hline Não realizou & 8 & 34,8 \\
\hline \multicolumn{3}{|c|}{ Resultados dos acidentados (Hep B) } \\
\hline Positivo & 0 & 0 \\
\hline Negativo & 23 & 100 \\
\hline \multicolumn{3}{|c|}{ Resultados dos acidentados (Hep C) } \\
\hline Positivo & 0 & 0 \\
\hline Negativo & 23 & 100 \\
\hline \multicolumn{3}{|c|}{ Resultados dos acidentados (HIV) } \\
\hline Positivo & 0 & 0 \\
\hline Negativo & 23 & 100 \\
\hline \multicolumn{3}{|c|}{ Resultados dos pacientes fonte (Hep $\mathrm{B}^{\star}$ ) } \\
\hline Positivo & 0 & 0 \\
\hline Negativo & 15 & 100 \\
\hline \multicolumn{3}{|c|}{ Resultados dos pacientes fonte $\left(\right.$ Hep $\mathrm{C}^{\star \star}$ ) } \\
\hline Positivo & 3 & 20 \\
\hline Negativo & 12 & 80 \\
\hline \multicolumn{3}{|c|}{ Resultados dos pacientes fonte (HIV) } \\
\hline Positivo & 2 & 13,3 \\
\hline Negativo & 13 & 86,7 \\
\hline \multicolumn{3}{|l|}{ Recomendações } \\
\hline $\mathrm{PEP}^{* * *}$ & 5 & 21,7 \\
\hline Reforço vacinal & 11 & 47,8 \\
\hline Nenhum procedimento & 7 & 30,4 \\
\hline
\end{tabular}

Legenda: *Hep B (Heptite B) ${ }^{* *}$ Hep C (Hepatite C) ${ }^{* * *}$ PEP (Profilaxia pós exposição).

Fonte: Galarça AMSS, et al., 2021.

Observa-se que, este é um fator limitante para o estudo, pois nem sempre o paciente relata a real condição sistêmica na anamnese. Além disso, com o restrito número amostral de notificações, não é possível correlacionar o uso de EPls com idade, tempo de graduação, condições sociodemográficas, entre outros aspectos. Dificuldade encontrada também em outros trabalhos que usaram de formulários para as entrevistas (OLIVEIRA AVM, et al., 2018). 
Percebe-se que na Odontologia há uma tendência de subnotificação dos acidentes perfurocortantes, especialmente entre os alunos (TREZENA S, et al., 2020). A literatura aponta aspectos que podem explicar tal fato, como burocracia, julgar que o ferimento seja de baixo risco, o medo de saber o estado sorológico, seu ou do paciente-fonte, a possível soroconversão, ou mesmo, falta de informação (LIMA AVM, et al., 2016; MUSSI M e MARASEA DCC, 2016).

Quando ocorre o acidente perfurocortante, o sangue pode entrar em contato com as mucosas, com locais onde a pele apresenta dermatites ou feridas abertas; assim como, lesões provocadas por instrumentos perfurantes ou cortantes (ex.: agulhas, lâminas de bisturi, vidrarias, etc.), exposições com risco de transmissão para hepatites virais e HIV (OLIVEIRA A, et al., 2018).

No entanto, a probabilidade de uma pessoa lesionada ser infectada por essas exposições depende da extensão da lesão, da quantidade de fluidos biológicos, da saúde do profissional e dos patógenos envolvidos do mesmo modo que, a condição clínica do paciente (fonte), e os procedimentos realizados após a exposição ao material biológico

Outra limitação do estudo é o curto tempo de levantamento dos dados, e pelo fato do protocolo Institucional ser atual e devido a isso, nem todos os alunos têm conhecimento deste. Quanto aos resultados dos exames para hepatites B e C nos acidentados, todos obtiveram testes negativos, ou seja, mesmo havendo acidentes perfurocortantes com material biológico contaminado, não houve infecção pelo vírus (GALARÇA AMSS e LUND RG 2020).

Apesar da alta incidência de infecção da Hep B, variando entre $6 \%$ e 30\%, sua capacidade de infectar é considerada alta com prevalência superior a $7 \%$, bem como $60 \%$ ou mais da população têm evidência sorológica de infecção prévia, felizmente existe vacina para prevenção, que além de ser segura e eficiente, possui grande cobertura populacional (GARBIN AJI, et al., 2016; NUNES AO, et al., 2016; ABREU CB, et al., 2021). O mesmo não ocorre para o vírus da Hepatite $C$, porém este possui baixa infectividade, cerca de $0,4 \%$ a $1,8 \%$, além de ter relação com a quantidade de sangue contaminado oriundo dos procedimentos, geralmente baixa (SOARES WKR, et al., 2018).

Da mesma forma, não houve nenhum caso de contaminação dos alunos por HIV. Isso pode ser explicado pela pequena quantidade de sangue envolvido em procedimentos odontológicos, e a probabilidade de infecção pelo HIV ser de $0,3 \%$ em acidentes percutâneos e 0,09\% em mucosas, aumentando conforme a profundidade das lesões e volume de sangue envolvido (WYŻGOWSKI P, et al., 2016; SARDEIRO TL, et al., 2019).

Dentre as recomendações, a profilaxia pós exposição (PEP), está indicada nas primeiras $72 \mathrm{~h}$ em caso de exposição ao HIV. É necessário reforçar a importância do esquema vacinal completo, além do exame AntiHBS, para confirmar que o profissional e/ ou estudante estejam imunes ao vírus da Hepatite B (GARBIN AJI, et al., 2016; GALARÇA AMSS, LUND RG 2020).

Nos casos em que o paciente-fonte não realizou o teste rápido ou os acidentados estavam com a vacinação adequada, não foi indicado nenhum procedimento pós exposição, apenas acompanhamento por 6 meses devido a janela imunológica (WYŻGOWSKI P, et al., 2016; GALARÇA AMSS, LUND RG 2020).

Como a grade curricular da FO contém significativa carga horária clínica, os alunos estão sujeitos aos riscos inerentes à profissão. Logo, é importante que estes sejam constantemente levados a refletir sobre as formas como ocorrem os acidentes, às doenças que podem estar expostos, como devem proceder e evitar tais situações. Dessa forma, ressalta-se a importância quanto ao registro das notificações para posterior divulgação das ocorrências (ARANTES DC, et al., 2015; MUSSI M, e MARASEA DCC, 2016; NOGUEIRA AS, et al., 2016).

É primordial que qualquer unidade de saúde possua um protocolo a ser seguido pelos profissionais em caso de acidentes, sendo necessário também um treinamento para toda a equipe. $O$ tempo decorrido entre $o$ momento do acidente e o início das medidas preventivas é um fator crítico para que não ocorram infecções (MARTINS MES, et al., 2020). Os acidentes com exposição a materiais biológicos devem ser tratados como casos de emergência médica (MINISTÉRIO DA SAÚDE, 2000; MARTINS JR, et al., 2018). 
A literatura recomenda que sejam adotados protocolos de registro, avaliação, aconselhamento, tratamento e acompanhamento dos indivíduos envolvidos em acidentes com agentes biológicos, e que a falta de um serviço destinado a esse fim na unidade, contribui para o agravamento dos casos (MARTINS JR, et al., 2018).

Com o propósito de reduzir os riscos de acidentes ocupacionais com material biológico nos ambientes de saúde, os norte-americanos, elaboraram um manual de condutas desenvolvido na Agência Federal Americana, Center for Disease Control and Prevention (CDC). Nele descrevam recomendações e formas de proceder frete a acidentes com materiais biológicos potencialmente contaminados, denominadas Precauções Padrão (PP), que devem ser seguidas pelos profissionais em ambiente de atendimento clínico, priorizando a saúde da equipe e dos pacientes (LIMA AVM, et al., 2016).

Atualmente, a FO conta com um serviço que atua a partir de um protocolo institucional aprovado para uso na Universidade. Não apenas registrando as ocorrências, mas prestando o auxílio necessário a quem procura atendimento (GALARÇA AMSS, e LUND RG, 2020).

Porém, percebe-se que por mais que haja ações de divulgação do serviço, ainda há casos de subnotificação. É preciso intensificar as campanhas de biossegurança de modo que os acadêmicos da FO sejam sensibilizados quanto a importância da previsibilidade e prevenção de acidentes (NOGUEIRA AS, et al., 2016; MAZZUTTI WJ, et al., 2018).

Com enfoque nas políticas públicas de saúde, as instituições de ensino devem estabelecer estratégias de intervenção para exposição das normas e protocolos, a fim de reduzir os riscos de acidentes. Dessa forma, a direção da instituição deve intervir junto ao serviço que presta atendimento aos alunos acometido por acidente perfurocortante e periodicamente expor os números de ocorrências, além dos locais e formas como estes corriqueiramente acontecem (NOGUEIRA AS, et al., 2016; OLIVEIRA A, et al., 2018).

É importante que todos os setores da saúde recebam orientações periódicas, pois quanto mais treinamento os profissionais recebem, menor é a estimativa de casos. Acredita-se que investir em campanhas de conscientização contribuem para a formação profissional dos acadêmicos, que logo estarão no mercado de trabalho e precisarão lidar com situações complexas de gestão de saúde (MUSSI M e MARASEA DCC, 2016; PAIVA SN, et al., 2017; MAZZUTTI WJ, et al., 2018).

A partir deste estudo, podem surgir novos trabalhos dando continuidade ao levantamento dos dados do serviço na FO, que com maior tempo de coleta, possibilitará a associação de fatores envolvidos nos acidentes perfurocortantes. Porém, para evitar subnotificações, sugere-se que periodicamente sejam feitas pesquisas com os alunos nas clinicas. E assim, identificar possíveis exposições a material biológico em um período de tempo. Dessa forma, o setor não depende apenas de o aluno procurar o serviço, mas vai ao encontro dessa categoria, diminuindo certas burocracias relatadas (GARBIN AJI, et al., 2016).

\section{CONCLUSÃO}

Avaliando os dados obtidos nesse estudo, percebe-se que existem casos de exposição a agentes biológicos na FO, sendo prevalente entre os acadêmicos. Estes acidentes representam riscos à saúde e devem ser tratados como emergência médica, a fim de reduzir possíveis complicações, visto que, muitos nem sempre relatam sua real condição sistêmica na anamnese, reforçando a importância do uso correto de EPIs em qualquer atendimento. Frente a isso, estimula-se a ação conjunta da direção da faculdade com o serviço que presta assistência aos acidentados por material biológico da instituição, no sentido de impulsionar e reforçar campanhas de conscientização à comunidade da FO, sobre normas e protocolos de biossegurança e notificação de acidentes, especialmente aos acadêmicos, expondo periodicamente os dados registrados no serviço.

\section{REFERÊNCIAS}

1. ARANTES DC, et al. Biossegurança aplicada à odontologia na Universidade Federal do Pará, cidade de Belém, estado do Pará, Brasil. Revista Pan-Amazônica de Saúde, 2015;6(1):8-8. 
2. ABREU CB, et al. Aspectos epidemiológicos da hepatite B no município de Teresina. Revista Eletrônica Acervo Saúde, 2021;13(5).

3. CARVALHO MJA, OLIVEIRA AHA. Equipamentos de proteção individual em odontologia: revisão integrativa de literatura. Revista Interface Saúde, 2015; 3(8): 1-6.

4. COUTO PLS, et al. Representações sociais acerca dos riscos de acidentes de trabalho. Revista Brasileira em Promoção da Saúde, 2018; $31(2): 70-74$.

5. CUNHA QB, et al. Fatores que interferem na adesão às precauções padrão por profissionais da saúde: revisão integrativa. Revista Enfermagem em Foco, 2017;8(1): 72-76.

6. FERREIRA LQ, et al. Hepatite B: conhecimento e atitudes de acadêmicos de Odontologia. R. Arch Health Invest, 2018; 7(7): 258-261.

7. GAMA TM, et al. Avaliação do nível de contaminação por bioaerossóis no ambiente clínico da Policlínica Odontológica da Universidade do Estado do Amazonas. Braz. J. of Develop. 2020; 6(6): 37397-37410.

8. GARBIN AJl, et al. Negligência no autocuidadoem saúde:a imunizaçãocontra a hepatite B na Odontologia. Archives of health investigation 2016;5(2): 85-89.

9. GALARÇA, AMSS, LUND RG. Protocolo pós-exposição ocupacional por material perfurocortante- UFPel- Faculdade de odontologia. $1^{\text {a }}$ ed. Pelotas: Editora UFPel, 50p.

10. LEÓN FC, et al. Magnitud del riesgo por accidentes con objetos cortopunzantes en la consulta odontológica. Revista Universitas Odontologica, 2019;38(80).

11. LIMA AVM, et al. Prevalência e fatores de risco de acidentes com materiais pérfuro-cortantes em alunos de graduação em odontologia. Braz Jor Periodontia, 2016;1(1):15-23.

12. MARTINS JR, et al. Percepção das Precauções Padrão, Prática do Reencape de Agulhas e Condutas Frente a Acidente com Material Biológico de Equipes de Saúde Bucal do Serviço Público Odontológico. Revista Ciência \& Trabalho, 2018;20(62): 70-75.

13. MARTINS MES, et al. Estudo dos acidentes com instrumentos perfurocortantes ecm clínica de graduação em odontologia. Revista da Faculdade de Odontologia de Porto Alegre, 2020;61(1): 61 -68.

14. MAZZUTTI WJ, et al. Nível de informação de estudantes de odontologia sobre riscos, prevenção e manejo de acidentes com perfurocortantes. Revista Rede Cuidados em Saúde, 2018;12(2): 17-27.

15. MINISTÉRIO DA SAÚDE. Guia Hepatites virais: o Brasil está atento Departamento de Vigilân cia Epidemiológica. Hepatites virais: o Brasil está atento.2008. Disponível em: https://bvsms.saude.gov.br/bvs/publicacoes/hepatites_virais_brasil_atento_3ed.pdf. Acessado em: 26 de junho de 2020.

16. MINISTÉRIO DA SAÚDE. Cartilha Serviços Odontológicos: prevenção e controle de riscos. Brasília: Ministério da Saúde. 2006. Disponível em: https://www.anvisa.gov.br/servicosaude/manuais/manual_odonto.pdf. Acessado em: 26 de junho de 2020.

17. MINISTÉRIO DA SAÚDE. Cartilha do Programa Nacional de Imunizações (PNI)40 anos. 2013. Disponível em https://bvsms.saude.gov.br/bvs/publicacoes/programa_nacional_imunizacoes_pni40.pd. Acessado em: 26 de junho de 2020.

18. MONTALLI VAM, et al. Individual biosafety barrier in dentistry: an alternative in times of covid-19. Preliminary study. Revista Gaúcha de Odontologia, 2020;(68) 1 p. 1-7.

19. MUSSI M, MARASEA DCC. A perspectiva da subnotificação de acidentes ocupacionais com dentistas. Revista Brasileira de Odontalgia, 2016;73(2):112-123.

20. NEDEL WL, SILVEIRA F. Different research designs and their characteristics in intensive care. Revista Brasileira de terapia intensiva, 2016;28(3): $256-267$.

21. NOGUEIRA SA, et al. Prevalência e notificações de acidentes de trabalho com exposição a material biológico na odontologia. Revista Ciência Plural, 2016;2(1): 102-119.

22. NUNES AO, et al. Vacinação contra hepatite $B$ em trabalhadores da saúde de um município da Bahia. Revista de saúde coletiva da UEFS, 2016; 5(1):9-16.

23. OLIVEIRA RHG, ALMEIDA, TF. Riscos Biológicos em Odontologia. Revista Baiana de Odontologia, 2015;6(1):34-46.

24. OLIVEIRA A, et al. Uso de equipamentos de proteção individual por cirurgiões dentistas em unidades básicas de saúde: estudo piloto. Revista Interface Saúde, 2018; 5(15), 64-70.

25. PAIVA SN, et al. Acidentes ocupacionais com material biológico em Odontologia: uma responsabilidade no ensino. Revista da ABENO, 2017; 17(3): 76-88.

26. PINHEIRO SL, et al. Influência da contaminação cavitária na adaptação marginal das restaurações de resina composta. Revista da Associação Paulista de Cirurgiões Dentistas, 2016;70 (10): 70-75.

27. SARDEIRO TL, et al. Acidente de trabalho com material biológico: fatores associados ao abandono do acompanhamento clínico-laboratorial. Revista da Escola de Enfermagem da USP, 2019; 53: 1-9. SOARES WKR, et al. Incidência de acidentes com perfurocortantes em profissionais de saúde em um hospital de grande porte na Amazônia Legal. Revista Brazilian Jourlnal of Healt Revil, 2018;1(1): 51-69.

28. TREZENA S, et al. Práticas em biossegurança frente aos acidentes ocupacionais entre profissionais da odontologia. Revista Arquivos em Odontologia, 2020;56(7): 1-8.

29. WYŻGOWSKI P, et al. Occupational HIV risk for health care workers: risk factor and the risk of infection in the course of professional activities. Rev Therapeut and clinic risk management, 2016;12(1): 989-994.

30. YOUNES T, et al. Biossegurança em Odontologia: o ponto de vista dos pacientes. Arquivos em Odontologia, $2017 ; 53$ $(14): 1-10$. 